\title{
Clear cell myomelanocytic tumour: minimally invasive treatment of a rare bladder tumour
}

\author{
Michael L. Pianezza, MD, MSc, FRCSC; ${ }^{*}$ Jack Slatnik, MD, FRCSC; ${ }^{\dagger}$ Howard J. Evans, MD, FRCSC
}

\begin{abstract}
Clear cell myomelanocytic tumours are extremely rare neoplastic growths considered to be members of the family of perivascular epithelioid cell tumours (PEComas), which have in common the coexpression of melanocytic and smooth muscle immunohistochemical markers. These tumours are known to be ubiquitous with uncertain tumour biology and to have unpredictable clinical behaviour. They have been reported in the genitourinary tract, including the kidney and prostate. There are only 3 reported cases of clear cell myomelanocytic tumours originating in the urinary bladder. We report a case of a 24-year-old woman with chronic pelvic pain who underwent laparoscopic partial cystectomy and total excision of a bladder mass. Pathological examination revealed primary PEComa of the urinary bladder. Subsequent follow-up procedures, including cystoscopy and imaging, have not revealed any evidence of disease recurrence. The patient remains clinically free of disease 3 months after surgery.
\end{abstract}

CUAJ 2008;2(2):230-4

\section{Introduction}

Clear cell myomelanocytic tumours are extremely rare and unusual mesenchymal neoplasms with an unpredictable natural history. These neoplasms belong to a group of perivascular epithelioid cell tumours (PEComas) with which they share the coexpression of melanocytic and smooth muscle immunohistochemical markers. ${ }^{1}$ This group of tumours also includes angiomyolipoma, lymphangioleiomyoma and clear cell "sugar" tumours. ${ }^{2-5}$ PEComas are occasionally associated with the tuberous sclerosis complex and can show loss of heterozygosity in the TSC1 and TSC2 region, or both. ${ }^{6}$ Both benign and malignant PEComas have been reported in diverse anatomic locations and are considered ubiquitous tumours. These tumours are often regarded as low-grade sarcomas and are treated as such.

There are several reports of PEComas arising in the genitourinary tract, including the kidneys and the prostate., Currently in the literature there are only 3 reported cases of PEComas originating in the urinary bladder. ${ }^{8-10}$ We report the diagnosis, management and short-term follow-up of the fourth documented case of primary PEComa of the urinary bladder. In addition, we report the first case of this tumour managed by partial cystectomy performed laparoscopically.

\section{Case report}

A 24-year-old otherwise healthy woman was referred to our hospital with a history of chronic pelvic pain. She did not complain of any urinary symptoms and had no history of gross hematuria. Her symptoms did not change with menstruation. She was a nonsmoker. Physical examination, including a pelvic examination, was unremarkable. The patient did not have any stigmata of tuberous sclerosis.

An ultrasound of the patient's pelvis was performed and revealed a solid right adnexal lesion measuring $2.9 \times 2.9 \times 2.8 \mathrm{~cm}$ just posterior to the bladder. The lesion appeared avascular and was protruding into the posterolateral aspect of the bladder wall. Magnetic resonance imaging (MRI) of the pelvis was subsequently performed and revealed a $3.0 \times$ $2.2-\mathrm{cm}$ fairly homogenous soft tissue mass within the right aspect of the bladder wall (Fig. 1). Part of the mass protruded into the bladder lumen, although most of the mass was exophytic. The mass demonstrated intermediate signal intensity on both $T_{1}$ and $T_{2}$ weighted images. Both the patient's ovaries and her uterus were normal on the MRI. The signal intensity did not favour an endometrioma.

The bladder mass prompted a cystoscopy,

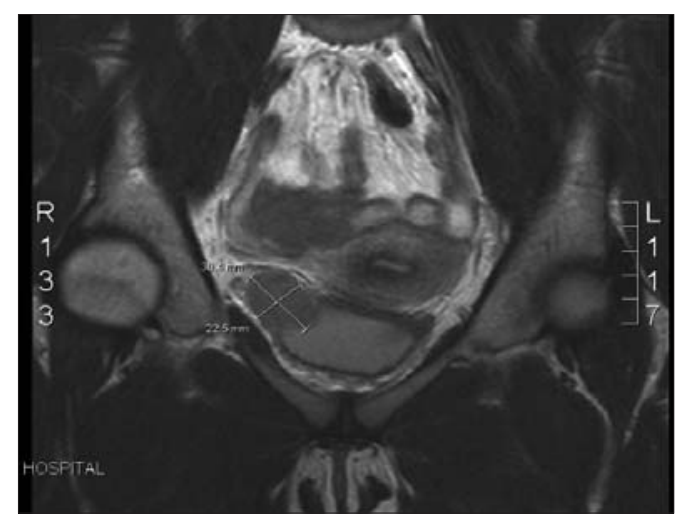

Fig. 1. Magnetic resonance imaging of the pelvis revealing a $3.0 \times 2.2-c m$ well-circumscribed oval-shaped soft tissue mass in the wall of the urinary bladder, on the right. 
which revealed a mucosal covered solid mass above the patient's right ureteric orifice. No other bladder lesions were identified. Her urethra was normal. Urinary cytology was negative for malignant cells. A cold cup biopsy of the bladder mass was performed and revealed only proteinaceous debris with no diagnostic cellular elements. Owing to the insignificant results obtained with cold cup biopsy during cystoscopy, a CT-guided fine needle aspiration and core biopsy of the bladder mass were performed. The results of the biopsy also revealed only proteinaceous debris with paucicellular smears.

The patient subsequently underwent laparoscopic partial cystectomy with complete excision of the bladder mass. An infrared fibreoptic ureteric stent was placed into the right ureter by cystoscopy before starting the laparoscopic procedure. The laparoscopic procedure involved the use of 4 ports. The ports included an optical port placed at the level of the umbilicus, two $5-\mathrm{mm}$ ports placed on the left side between the anterior superior iliac spine (ASIS) and the umbilicus, and one 12-mm port placed on the right side between the ASIS and the umbilicus. The peritoneum around the bladder was initially incised to gain access to Retzius cavity. The bladder was completely mobilized. A cystotomy was made. The mass was identified just above the right ureteric orifice. The mass was circumferentially removed from the bladder, leaving the mucosa over top of it. The mass was placed into an Endocatch bag (Ethicon Endo-Surgery Inc., Cincinnati, Ohio). Both the anterior and posterior walls of the bladder were closed with 2.0 Vicryl (EthiconEndo-Surgery Inc., Cincinnati, Ohio) in a running continuous fashion. The 2 sutures were tied together in the middle. The bladder was filled with saline and no leak was identified. The fibreoptic ureteric stent was removed and exchanged for a 6 -French $\times 24-\mathrm{cm}$ double J ureteric stent. A Jackson-Pratt drain was placed.

The patient's postoperative course was unremarkable. She was discharged home following 2 days in hospital. A cystogram was performed 1 week postoperatively and it confirmed there was no bladder leak. The foley catheter was subsequently removed. The right ureteric stent was removed 2 weeks following surgery. Pathology results revealed a clear cell myomelanocytic tumour of the urinary bladder (PEComa). Surgical margins were negative. Three months after surgery, the patient had no evidence of clinical recurrence and had a resolution of her pelvic pain.

\section{Pathology}

Gross examination of the bladder mass revealed a well-circumscribed piece of firm tissue measuring $3.4 \times 2.5 \times 1.8 \mathrm{~cm}$. The mass had a slightly brownish discolouration. Microscopic examination revealed the mass was formed of mainly spindle cells. The lesion demonstrated mostly pushing but occasionally infiltrative margins. The cells were arranged in loosely cohesive fascicles with hyalinized fibrous tissue between the fascicles. No necrosis was seen. The tissue surrounding the lesion consisted of smooth muscle with focally seen overlying benign urothelium. The cells were monomorphic with moderate cytological atypia. The cytoplasm was eosinophilic or clear. Mitotic activity of 1 mitosis per 50 high-power field was present. The tumour extended through the entire thickness of the bladder wall and focally was seen abutting perivesical adipose tissue. There was no lymphovascular space involvement. Surgical margins were negative (Fig. 2).

The tumour was further investigated with immunostains. The tumour cells were HMB45, S100, caldesmon and actin positive (Fig. 3). MART-1, CD34, K903, pankeratin, calretinin, CD99, ALK-1, C-Kit and desmin staining were negative. The PAS was positive and the PASD was negative.

The light microscopic picture combined with the immunohistochemistry result was diagnostic for clear cell myomelanocytic tumour.

\section{Discussion}

The World Health Organization defines PEComas as "mesenchymal tumors composed of histologically and immunohistochemically distinctive perivascular epithelioid cells." 11 These tumours have in common the presence of epithelioid to spindle cells with eosinophilic to clear cytoplasm, which demonstrate positive immunostaining for markers of both myoid (smooth muscle actin, desmin) and melanocytic (HMB-45) differentiation. The PEComa family of tumours includes tumours such as angiomyolipoma, clear cell "sugar" tumour and lymphangioleiomyomatosis. ${ }^{2-5}$ In addition, the PEComa family consists of a number of unusual 
visceral, intra-abdominal, soft tissue and bone tumours, which have been described under a variety of names, including "clear cell myomelanocytic tumor of the falciform ligament/ligamentum teres," "abdominopelvic sarcoma of perivascular epithelioid cells" and "primary extrapulmonary sugar tumor," among others. ${ }^{6}$ PEComas have been documented in a variety of anatomical locations, including the pancreas, small and large intestine, ligamentum teres/falciform ligament, common bile duct, kidney, bladder, prostate, breast, uterus, cervix, vulva, ovary, broad ligament, heart, base of skull and soft tissue. ${ }^{8,12-15}$
The major differential diagnosis of this tumour around the anatomical site of the urinary bladder, includes smooth muscle tumours. Smooth muscle tumours, including leiomyoma and leiomyosarco$\mathrm{ma}$, are the most common mesenchymal tumours of the urinary bladder in adults. ${ }^{16}$ The cytoplasm of the neoplastic smooth muscle cells is usually more eosinophilic rather than the clear-to-pale cytoplasm in the tumour cells of PEComas. Both smooth muscle tumours and PEComas are known to express actin, however the smooth muscle tumour has not been reported to be immunoreactive for melanocytic markers. The strong

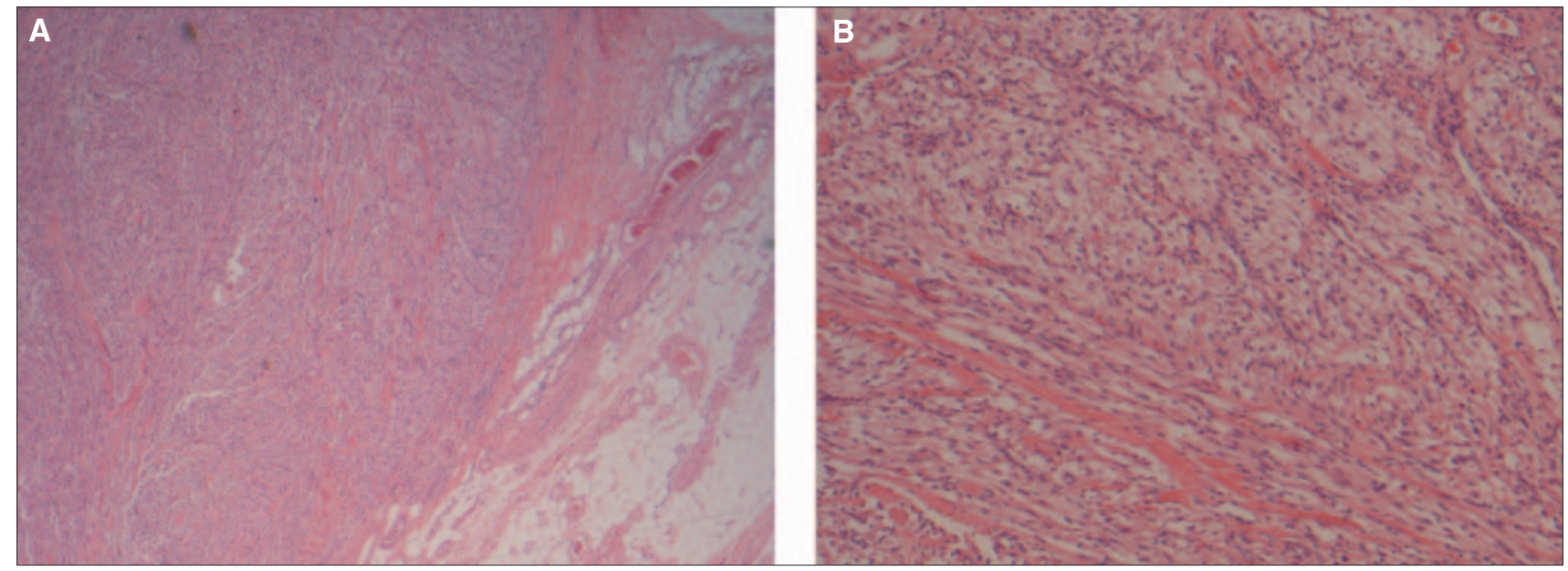

Fig. 2. Histological examination at low magnification demonstrating the clear boundary of the mass with the bladder wall (A) (hematoxylin-eosin stain, original magnification $100 \times$ ). The tumour extends through the entire thickness of the bladder wall and focally is seen abutting the perivesical adipose tissue. High magnification of the mass demonstrating the eosinophilic spindled cells arranged in loosely cohesive fascicles (B) (hematoxylin-eosin stain, original magnification $200 \times)$.
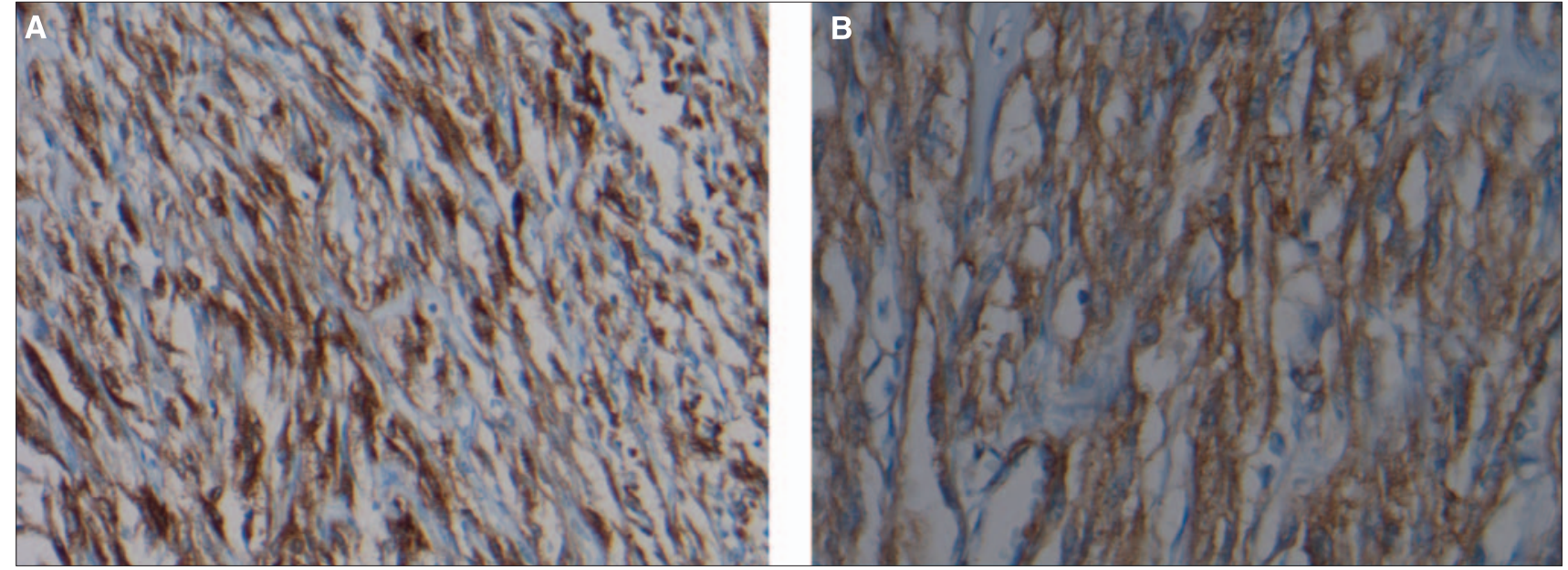

Fig. 3. Histological examination of the tumour cells demonstrating strong and diffuse positivity for immunoperoxidase HMB-45 (A) (original magnification $200 \times$ ) and smooth muscle actin (B) (immunoperoxidase stain, original magnification $400 \times$ ). 
immunoreactivity for HMB-45 in the present case can essentially rule out the possibility of a smooth muscle tumour.

Additional differential diagnoses to consider would be malignant melanoma, postoperative spindle cell tumour and sarcomatoid carcinoma. Each of these possibilities can be easily excluded on the basis of morphology and immunohistochemistry.

Only 3 other cases of primary PEComa involving the urinary bladder have been described before ours. The first report by Pan and colleagues described a 33-year-old Taiwanese woman who was found to have a PEComa of the bladder during a workup for dysmenoorhea. ${ }^{8}$ The patient did not have any lower urinary tract symptoms or gross hematuria. She underwent partial cystectomy with total excision of the tumour and remained without evidence of disease with 6 years of follow-up. Kalyanasundaram and colleagues reported the case of a 19-year-old woman who presented with gross hematuria and was found to have an intraluminal, polypoidal pedunculated mass in her bladder. ${ }^{9}$ She underwent transurethral resection of the bladder mass, which revealed primary PEComa of the bladder on pathological evaluation. The patient was later lost to follow-up. Parfitt and coauthors described a 48-year-old man who presented with dysuria, passage of urinary sediment and lower abdominal discomfort. ${ }^{10}$ The patient underwent laparotomy, partial cystectomy and partial small bowel resection for a presumed enterovesical fistula. Pathological evaluation revealed primary PEComa of the bladder. The patient subsequently underwent adjuvant interferon (IFN)- $\alpha$ immunotherapy. He remained without evidence of disease recurrence 48 months following surgery.

PEComas demonstrate uncertain tumour biology and unpredictable clinical behaviour. The majority of reported PEComas behaved in a benign fashion; however, a minority have demonstrated malignant behaviour with locally destructive recurrences, distant metastasis and death..$^{7,12,13}$ One of the largest series of cases published in the literature included 26 patients with PEComas of soft tissue and gynecologic origin. ${ }^{17}$ Of the 26 patients, clinical follow-up data was available for 24 patients, with a median of 30 months. Three patients demonstrated local recurrence and 5 showed distant metastases. At the time of final clinical follow-up,
2 patients ( $8 \%$ ) had died of disease, 4 patients $(17 \%)$ were alive and had metastatic or unresectable local disease and 18 patients (75\%) were alive with no evidence of disease. This paper also included a review of the literature combining their results of 24 cases with 45 previously reported cases of PEComa, with special attention to features predictive of clinical behaviour. They found that recurrence or metastasis (or both) were strongly associated with tumour size $>5 \mathrm{~cm}$, infiltrative growth pattern, high nuclear grade, necrosis and a mitotic index of $>1$ per $50 \mathrm{HPF}$.

The optimal treatment of PEComa is not known at this time. Surgical excision is the mainstay of therapy as most tumours are benign. For the minority of patients with locally advanced or metastatic disease, therapies incorporating radiation, chemotherapy and immunotherapy have been reported. Locally advanced and metastatic disease generally portends a poor prognosis.

\section{Conclusion}

In summary, we report the fourth documented case of primary PEComa of the urinary bladder. In addition, we report the first case of this tumour treated with partial cystectomy performed laparoscopically. No adjuvant therapy was given. Subsequent follow-up procedures have not revealed any evidence of disease recurrence 3 months after surgery.

From the Royal Alexandra Hospital, *Division of Urology, Department of Surgery, and †Department of Pathology, University of Alberta, Edmonton, Alta.

This article has been peer reviewed.

Competing interests: None declared.

\section{References}

1. Bonetti F, Pea M, Martignoni G, et al. PEC and sugar. Am I Surg Pathol 1992;16:307-8.

2. Dalle I, Sciot R, de Vos R, et al. Malignant angiomyolipoma of the liver: a hitherto unreported variant. Histopathology 2000;36:443-50.

3. Eble JN, Amin MB, Young RH. Epithelioid angiomyolipoma of the kidney: a report of five cases with a prominent and diagnostically confusing epithelioid smooth muscle component. Am J Surg Pathol 1997;21:1123-30.

4. Bonetti F, Pea M, Martignoni G, et al. Clear cell ('sugar') tumour of the lung is a lesion strictly related to angiomyolipoma: the concept of a family of lesions characterized by the presence of the perivascular epithelioid cells (PEC). Pathology 1994;26:230-6.

5. Chan JK, Tsang WY, Pau MY, et al. Lymphangiomyomatosis and angiomypolipoma: closely related entities characterized by hemartomatous proliferation of HMB-45-positive smooth muscle. Histopathology 1993;22:445-55.

6. Bonetti F, Pea M, Martignoni $G$, et al. The perivascular epithelioid cell and related lesions. Adv Anat Pathol 1997:4:343-58. 
7. Pan CC, Yang AH, Chiang H. Malignant perivascular epithelioid cell tumour ('PEComa') involving the prostate. Arch Pathol Lab Med 2003;127:e96-8.

8. Pan CC, Yu IT, Yang AH, et al. Clear cell myomelanocytic tumour of the urinary bladder. Am I Surg Pathol 2003;27:689-92.

9. Kalyanasundaram K, Parameswaran A, Mani R. Perivascular epithelioid tumour of urinary bladder and vagina. Ann Diagn Pathol 2005;9:275-8.

10. Parfitt JR, Bella A, Wehrli BM, et al. Primary PEComa of the bladder treated with primary excision and adjuvant interferon-alpha immunotherapy: a case report. BMC Urol 2006;6:20.

11. Folpe AL. Neoplasms with perivascular epithelioid cell differentiation (PEComas). In: Fletcher CDM, Unni KK, Mertens F, editors. World Health Organization classification of tumours: pathology and genetics of tumours of soft tissue and bone. Lyon: IARC Press; 2002. p. 221-22.

12. Harris GC, McCulloch TA, Perks G, et al. Malignant perivascular epithelioid cell tumour ("PEComa") of soft tissue: a unique case. Am I Surg Pathol 2004;28:1655-8.

13. Lehman NL. Malignant PEComa of the skull base. Am I Surg Pathol 2004;28:1230-2.
14. Sadeghi S, Krigman H, Maluf H. Perivascular epithelioid clear cell tumour of the common bile duct. Am I Surg Pathol 2004;28:1107-10.

15. Evert $M$, Wardelmann $E$, Nestler $G$, et al. Abdominopelvic perivascular epithelioid cell sarcoma (malignant PEComa) mimicking gastrointestinal stromal tumour of the rectum. Histopathology 2005:46:115-7.

16. Martin SA, Sears DL, Sebo TJ, et al. Smooth muscle neoplasms of the urinary bladder: a clinicopathologic comparison of leiomyoma and leiomyosarcoma. Am I Surg Pathol 2002;26:292-300

17. Folpe AL, Mentzel T, Lehr HA, et al. Perivascular epithelioid cell neoplasms of soft tissue and gynecologic origin: a clinicopathologic study of 26 cases and review of the literature. Am J Surg Pathol 2005;29:1558-75.

Correspondence: Dr. Howard J. Evans, Assistant Clinical Professor, Division of Urology, Department of Surgery, University of Alberta, Hys Medical Centre, 40011010101 St NW, Edmonton AB T5H 4B9; hevans@aburologyinstitute.com

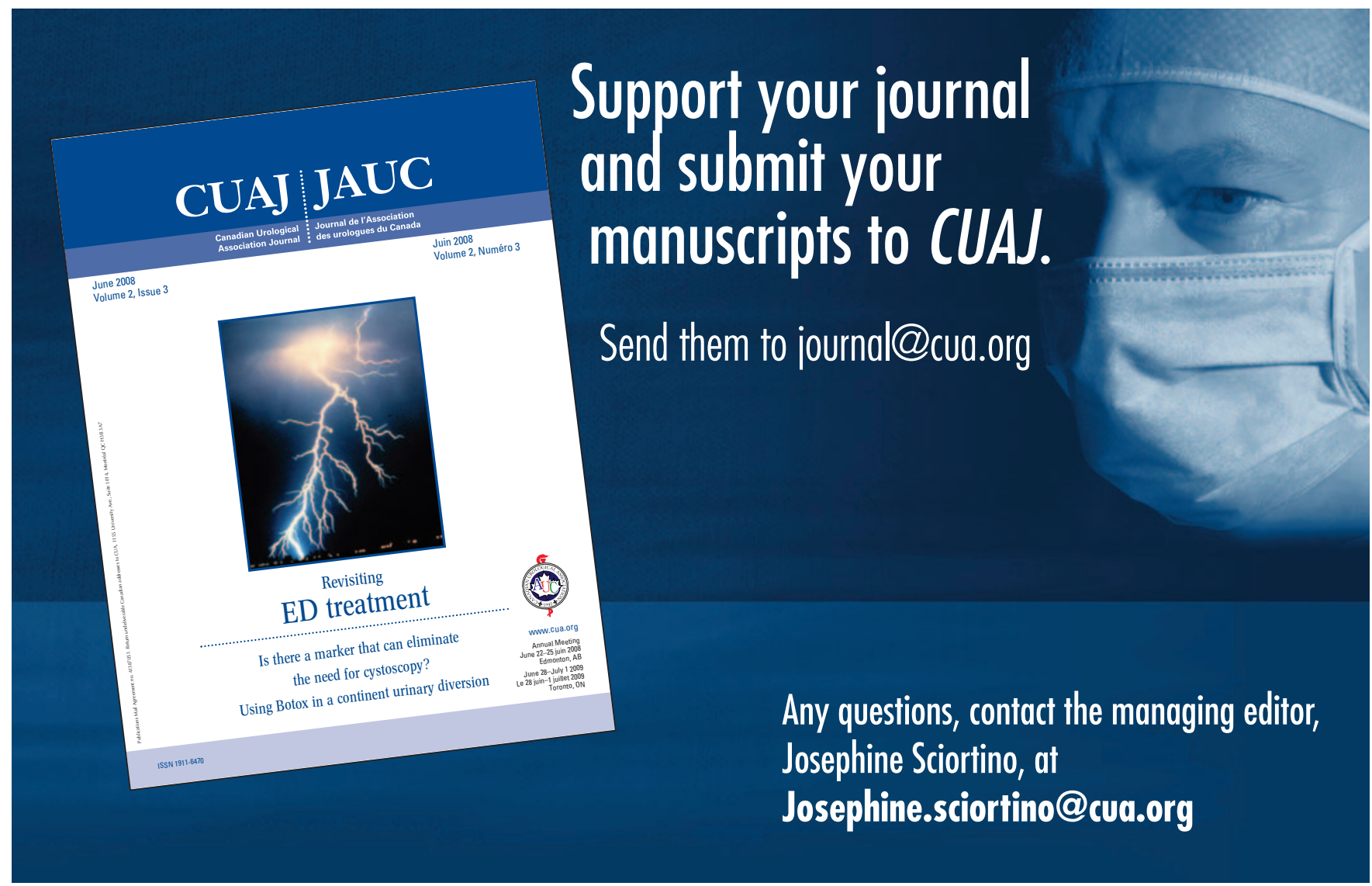

